doi:10.13108/2020-12-4-3

\title{
ON INFINITE SYSTEM OF RESONANCE AND EIGENVALUES WITH EXPONENTIAL ASYMPTOTICS GENERATED BY DISTANT PERTURBATIONS
}

\author{
D.I. BORISOV, M.N. KONYRKULZHAEVA
}

\begin{abstract}
We consider an one-dimensional Schrödinger operator with four distant potentials separated by large distance. All distances are proportional to a sam large parameter. The initial potentials are of kink shapes, which are glued mutually so that the final potential vanishes at infinity and between the second and third initial potentials and it is equal to one between the first and the second potentials as well as between the third and fourth potentials. The potentials are not supposed to be real and can be complex-valued. We show that under certain, rather natural and easily realizable conditions on the four initial potentials, the considered operator with distant potentials possesses infinitely many resonances and/or eigenvalues of form $\lambda=k_{n}^{2}, n \in \mathbb{Z}$, which accumulate along a given segment in the essential spectrum. The distance between neighbouring numbers $k_{n}$ is of order the reciprocal of the distance between the potentials, while the imaginary parts of these quantities are exponentially small. For the numbers $k_{n}$ we obtain the representations via the limits of some explicitly calculated sequences and the sum of infinite series. We also prove explicit effective estimates for the convergence rates of the sequences and for the remainders of the series.
\end{abstract}

Keywords: resonance, exponential asymptotics, distant perturbations, non-self-adjoint operator

Mathematics Subject Classification: 34L05, 34D10, 34E10

\section{INTRODUCTION}

Problems with distant perturbations have a rich history and are studied rather intensively. A classical example of an operator with distant perturbations is the Schrödinger operator on the axis with a pair of distant potentials. A more general formulation involves elliptic differential operators in unbounded periodic domains with coefficients containing several localized profiles. These profiles are connected by some periodic background; on different connections the structure of such background can be different. By nowadays, the behavior of the resolvents and isolated eigenvalues was studied quite well and results of general nature were obtained in [2], 3], [10], [11, [17], [18]; earlier results for particular models can be found in classical works [5], [15], [16], [19], [20], [22], 23].

The behavior of the resonances of the operators with distant perturbations is studied much less. For a classical case of the Schrödinger operator in the three-dimensional space with several distant perturbations the behavior of the resonances was studied in [21]. A one-dimensional case with a pair of general distant localized perturbations, not necessarily described by symmetric operators, was considered in recent works [12], [13]. We mention a similar one-dimensional model with a truncated periodic potential [6], as well as its discrete analogue [24]. It was found in the cited works that as the distance between the supports of the distant perturbations grows,

D.I. Borisov, M.N. Konyrkulzhaeva, On infinite System of REsonances And Eigenvalues With EXPONENTIAL ASYMPTOTICS GENERATED BY DISTANT PERTURBATIONS.

(C) Borisov D.I.,Konyrkulzhaeva M.N. 2020.

Submitted August 25, 2020. 
in the vicinity of the bottom of the essential spectrum of the considered operator, infinitely many resonances could arise and these resonances accumulated along some curves. The distance from these resonances to the essential spectrum was of order of the reciprocal of the distance between the distant perturbations.

In paper [9], there was studied a problem for the Laplacian in the strip, where the distant perturbations were replaced by the Dirichlet and Neumann boundary conditions. A similar problem but in a multi-dimensional cylinder was studied in [1]. In was shown in these two works that the eigenvalues embedded into the essential spectrum, bifurcated into finitely many resonances with exponentially small asymptotics. In a very recent work [14] this phenomenon was found in the general case for an arbitrary elliptic operator with finitely many distant perturbations in a multi-dimensional cylinder.

In all studied models, in the case, when the resonances and/or eigenvalues emerged and accumulated along some segment in the essential spectrum, their asymptotics were also power with respect to the distance between the distant potentials [6, [12, [13], 21], [24]. In the case of perturbing the eigenvalues embedded into the essential spectrum, only finitely many resonances with exponential asymptotics arose [1], 9], 14]. This poses a natural question: whether this is true that the emergence of infinitely many resonances means that they always have power in the distance asymptotics, while in the case of emergence of finitely many resonances the corresponding asymptotics are always exponential? In the present work we disprove this conjecture providing an appropriate example. Namely, we consider a one-dimensional Schrödinger operator with four distant potentials. The shapes of the potentials are of kink type, which are glued so that the final potential vanishes at infinity and between the second and third potentials and it is equal to one between the first and second potentials as well as between the third and the fourth potentials, see Figures 1 and 2. The potentials are not assumed to be necessarily real-valued, and can be also complex-valued. This is why, in the vicinity of the essential spectrum, not only resonances can emerge, but also eigenvalues.

Our main result is as follows. Under certain, rather natural and easily realizable conditions for the four initial potentials, the operator with distant perturbations can have infinitely many resonances and/or eigenvalues of form $\lambda=k_{n}^{2}, n \in \mathbb{Z}$, which accumulate along some segment in the essential spectrum. The distance between the neighbouring numbers $k_{n}$ is a quantity of order a power of the distance between the potentials, while the imaginary parts of these quantities are exponentially small. On the base of the techniques of work [13], we obtain representations for the numbers $k_{n}$ via the limits of some explicitly calculated sequences and the sums of some infinite series. We also prove explicit effective estimates for the convergence rate of the sequence and for the remainders of the series.

\section{MODEL AND RESUlts}

Let $x \in \mathbb{R}$ be a real variable, $V_{i}=V_{i}(x), i=1, \ldots, 4$, be piece-wise complex-valued potentials defined on the real axis and satisfying the conditions

$$
\begin{array}{lllll}
V_{i}(x)=0 \quad \text { as } \quad x<-x_{0}, & V_{i}(x)=1 \quad \text { as } \quad x>x_{0}, & i=1,3, \\
V_{i}(x)=1 \quad \text { as } \quad x<-x_{0}, & V_{i}(x)=0 \quad \text { as } \quad x>x_{0}, & i=2,4,
\end{array}
$$

where $x_{0}$ is some fixed positive number. By $\ell$ we denote a large positive parameter, while $\alpha_{ \pm}$ are some fixed positive numbers. We define one more potential:

$$
V_{\ell}(x):= \begin{cases}V_{1}\left(x+\left(2 \alpha_{-}+1\right) \ell\right) & \text { as } \quad x<-\left(\alpha_{-}+1\right) \ell, \\ V_{2}(x+\ell) & \text { as }-\left(\alpha_{-}+1\right) \ell<x<0, \\ V_{3}(x-\ell) & \text { as } 0<x<\left(\alpha_{+}+1\right) \ell, \\ V_{4}\left(x-\left(2 \alpha_{+}+1\right) \ell\right) & \text { as } \quad x>\left(\alpha_{+}+1\right) \ell .\end{cases}
$$



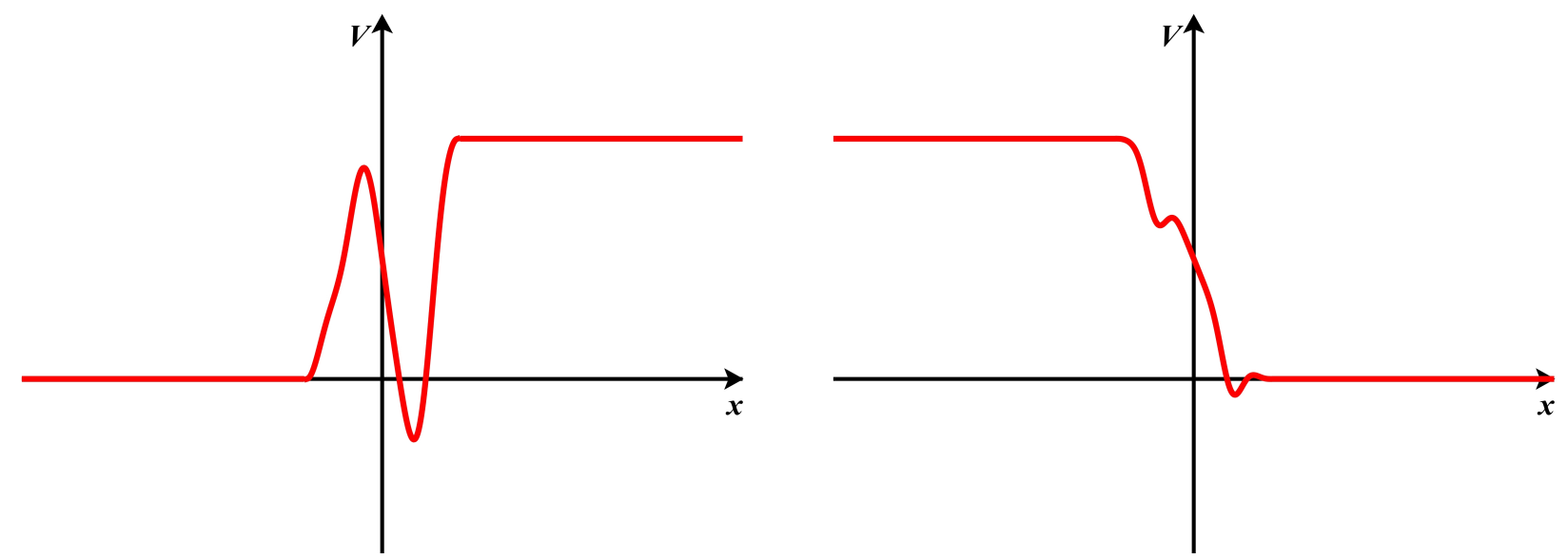

FiguRE 1. Sketched graphs of the potentials $V_{1}, V_{3}$ (left figure) and of the potentials $V_{2}, V_{4}$ (right figure).

A sketched graph of the potentials $V_{i}$ and $V_{\ell}$ is shown on Figures 1, 2. A main feature of the potential $V_{\ell}$ is that as the parameter $\ell$ grows, the intervals, on which the function $V_{\ell}$ move away one from another, while the lengths of the segments, on which the potential $V_{\ell}$ is equal to zero or one, increase.

In this work we consider a Schrödinger operator on the axis with the complex-valued potential $V_{\ell}$ :

$$
\mathcal{H}_{\ell}:=-\frac{d^{2}}{d x^{2}}+V_{\ell}
$$

This operator is considered as unbounded in the space $L_{2}(\mathbb{R})$ on the domain $W_{2}^{2}(\mathbb{R})$. It is obviously closed and in the case of a real-valued potential $V_{\ell}$ it is also self-adjoint. The main aim of the work was the behavior of the resonances and eigenvalues of this operator as $\ell \rightarrow+\infty$.

We define the resonances and the eigenvalues of the operator $\mathcal{H}_{\ell}$ as values $\lambda=k^{2}, k \in \mathbb{C}$, for which the equation

$$
\left(-\frac{d^{2}}{d x^{2}}+V_{\ell}\right) \psi=\lambda \psi \quad \text { in } \quad \mathbb{R}
$$

possesses a non-trivial solution with the following behavior at infinity:

$$
\psi(x)=c_{ \pm} e^{ \pm \mathrm{i} k x}, \quad x \rightarrow \pm \infty,
$$

where $c_{ \pm}$are some constants. The latter identities obviously fit the definition of the potential $V_{\ell}$ since the latter, by definition, vanishes as $x<-\alpha_{-} \ell-x_{0}$ and $x>\alpha_{+} \ell+x_{0}$. For $\operatorname{Im} k>0$, relations (2.3) describe an exponentially decaying at infinity solution to equation (2.2). This solution is an eigenfunction associated with an eigenvalue $\lambda=k^{2}$. If $\operatorname{Im} k \leqslant 0$, relations (2.3) describe either an exponentially growing or a non-decaying oscillating at infinity solution and in this case the quantity $\lambda=k^{2}$ is a resonance. We also mention that the above definition of the resonance is equivalent to defining it as a pole of an appropriate meromoprhic continuation of the resolvent through the essential spectrum [25].

To formulate the main results, we shall need some auxiliary notations. We begin with defining of Jost-like functions for the potentials $V_{i}, i=1, \ldots, 4$. Namely, by $X_{i}=X_{i}(x, k), i=1, \ldots, 4$, we denote the solutions of the equations

$$
\left(-\frac{d^{2}}{d x^{2}}+V_{j}(x)-k^{2}\right) X_{j}=0 \quad \text { in } \quad \mathbb{R}
$$




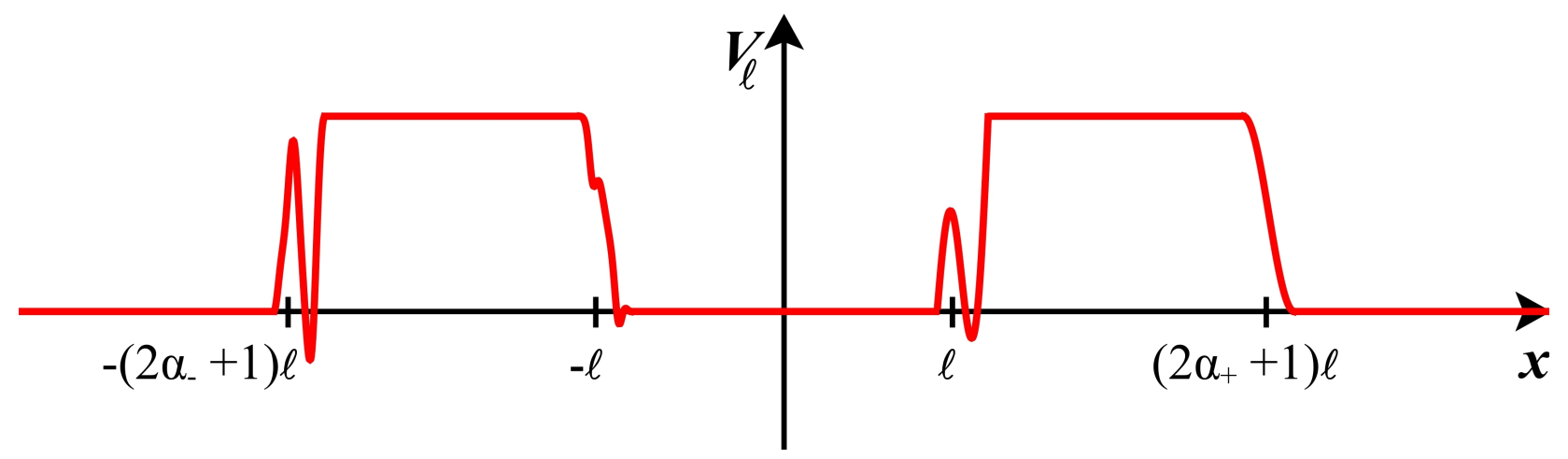

Figure 2. Sketched graphs of the potentials $V_{\ell}$.

with the following behavior at infinity:

$$
\begin{array}{ll}
X_{j}(x, k)=e^{-\mathrm{i} k x} & \text { as } \quad x<-x_{0}, \\
X_{j}(x, k)=a_{j}(k) e^{-\sqrt{1-k^{2}} x}+b_{j}(k) e^{\sqrt{1-k^{2}} x} & \text { as } \quad x>x_{0},
\end{array}
$$

for $j=1,3$, and

$$
\begin{array}{ll}
X_{j}(x, k)=e^{\mathrm{i} k x} & \text { as } \quad x>x_{0}, \\
X_{j}(x, k)=a_{j}(k) e^{-\sqrt{1-k^{2}} x}+b_{j}(k) e^{\sqrt{1-k^{2}} x} & \text { as } \quad x<-x_{0},
\end{array}
$$

for $j=2,4$. Here $a_{j}=a_{j}(k), b_{j}=b_{j}(k)$ are some functions and the branch of the root is fixed by the condition $\sqrt{1}=1$. A complex parameter $k$ is supposed to range in the complex plane with two cuts along the real semi-axes, namely,

$$
k \in \Xi:=\mathbb{C} \backslash\{k: \pm k \in[1,+\infty)\} .
$$

We denote:

$$
f(k):=\frac{a_{2}(k) b_{3}(k)}{a_{2}(-k) b_{3}(-k)} .
$$

Throughout the work we assume the following condition: there exists a segment $\left[\beta_{-}, \beta_{+}\right] \in$ $(-1,1)$ such that the inequalities hold:

$$
a_{2}(-k) \neq 0, \quad b_{1}(k) \neq 0, \quad b_{3}(-k) \neq 0, \quad a_{4}(k) \neq 0 \quad \text { as } \quad k \in\left[\beta_{-}, \beta_{+}\right]
$$

and the identity

$$
|f(k)|=1 \quad \text { as } \quad k \in\left[\beta_{-}, \beta_{+}\right]
$$

We let:

$$
\varkappa_{n}(\ell):=\frac{\pi n}{2 \ell}, \quad n \in \mathbb{Z}
$$

By $h^{[m]}$ we denote an $m$-multiple superposition of the function $h$, that is, $h^{[m]}:=\underbrace{h \circ h \circ \cdots \circ h}_{m \text { times }}$, where the symbol $\circ$ denotes the superposition of the functions, namely, $h \circ g=h(g)$.

We define an essential spectrum of the operator $\mathcal{H}_{\ell}$ in terms of the characteristic sequences, that is, a complex number $\lambda$ is in the essential spectrum $\sigma_{\text {ess }}\left(\mathcal{H}_{\ell}\right)$ of the operator $\mathcal{H}_{\ell}$ if there exists a sequence $u_{n}$ in the domain of this operator, bounded and non-compact in $L_{2}(\mathbb{R})$ such that $\left(\mathcal{H}_{\ell}-\lambda\right) u_{n} \rightarrow 0$ as $n \rightarrow+\infty$. It is obvious that under such definition, the essential spectrum of the operator $\mathcal{H}_{\ell}$ coincides with the negative semi-axis:

$$
\sigma_{\text {ess }}\left(\mathcal{H}_{\ell}\right)=[0,+\infty) \text {. }
$$

In the fourth section we shall prove the following auxiliary lemma. 
Lemma 2.1. Assume that conditions (2.7), (2.8) hold. There exists a simply-connected neighbourhood $\Omega$ of the segment $\left[\beta_{-}, \beta_{+}\right]$in the complex plane such that the function

$$
h(k):=-\frac{\mathrm{i}}{4} \ln f(k)
$$

is univalent and holomorphic in $k \in \bar{\Omega}$ and is real as $k \in\left[\beta_{-}, \beta_{+}\right]$. For sufficiently large $\ell$ the equation

$$
e^{4 \mathrm{i} k \ell}=f(k)
$$

possesses a series of roots $k=K_{n}(\ell)$, where an integer parameter $n$ ranges over the set $M_{\ell}$,

$$
M_{\ell}:=\left\{n \in \mathbb{Z}: \varkappa_{n}(\ell) \in I_{\ell}\right\}, \quad I_{\ell}:=\left[\beta_{-}-\ell^{-1} h\left(\beta_{-}\right), \beta_{+}-\ell^{-1} h\left(\beta_{+}\right)\right] .
$$

These roots are represented by the limits

$$
K_{n}(\ell)=\varkappa_{n}(\ell)+\lim _{m \rightarrow+\infty} h_{n}^{[m]}(0, \ell), \quad h_{n}(k, \ell):=\ell^{-1} h\left(k+\varkappa_{n}(\ell)\right),
$$

and by the sums of the series

$$
K_{n}(\ell)=\varkappa_{n}(\ell)+\sum_{m=1}^{\infty} \frac{1}{m ! \ell^{m}} \frac{d^{m-1} h^{m}}{d k^{m-1}}\left(\varkappa_{n}(\ell)\right)=\sum_{m=1}^{\infty} \frac{1}{m ! \ell^{m}} \frac{d^{m-1}\left(\varkappa_{n}(\ell)+h\right)^{m}}{d k^{m-1}}(0) .
$$

The estimates hold:

$$
\begin{aligned}
& \left|K_{n}(\ell)-\varkappa_{n}(\ell)-h_{n}^{[m]}(0, \ell)\right| \leqslant M\left(M^{\prime}\right)^{m} \ell^{-m-1} \\
& \begin{aligned}
& \mid K_{n}(\ell)-\varkappa_{n}(\ell)-\sum_{m=1}^{p} \frac{1}{m ! \ell^{m}} \frac{d^{m-1} h^{m}}{d k^{m-1}}\left(\varkappa_{n}(\ell) \mid\right. \leqslant \frac{1}{(p+1) ! \ell^{p+1}} \max _{k \in \bar{\Omega}_{\ell}}\left|\frac{d^{p} h^{p+1}}{d k^{p}}(k)\right| \\
& \leqslant \frac{|\partial \Omega|}{2 \pi(p+1)}\left(\frac{M}{\rho \ell}\right)^{p+1}, \\
&\left|K_{n}(\ell)-\sum_{m=1}^{p} \frac{1}{(p+1) ! \ell^{p+1}} \frac{d^{m-1}\left(\varkappa_{n}(\ell)+h\right)^{m}}{d k^{m-1}}(0)\right| \leqslant \frac{1}{(p+1) ! \ell^{p+1}} \frac{\max }{\Omega_{\ell}}\left|\frac{d^{p}}{d k^{p}}\left(\varkappa_{n}(\ell)+h(k)\right)^{p+1}\right| \\
& \leqslant \frac{|\partial \Omega|}{2 \pi(p+1)}\left(\frac{M+\beta+1}{\rho \ell}\right)^{p+1},
\end{aligned}
\end{aligned}
$$

where we have denoted

$$
\begin{gathered}
M:=\max _{k \in \bar{\Omega}}|h(k)|, \quad M^{\prime}:=\max _{k \in \bar{\Omega}}\left|h^{\prime}(k)\right|, \\
\rho:=\operatorname{dist}\left(\partial \Omega,\left[\beta_{-}, \beta_{+}\right]\right)-M \ell^{-1}, \quad \beta:=\max \left\{\left|\beta_{-}\right|,\left|\beta_{+}\right|\right\}, \quad \alpha:=\min \left\{\alpha_{-}, \alpha_{+}\right\} .
\end{gathered}
$$

Let $N(\ell)$ be the number of the elements in the set $M_{\ell}$ and

$$
\begin{gathered}
\varkappa_{\text {min }}(\ell):=\min _{n \in M_{\ell}} \varkappa_{n}(\ell), \quad \varkappa_{\text {max }}(\ell):=\max _{n \in M_{\ell}} \varkappa_{n}(\ell), \\
\Pi_{\ell}:=\left\{k \in \Omega: \varkappa_{\text {min }}(\ell)-\frac{\pi}{4 \ell} \leqslant \operatorname{Re} k \leqslant \varkappa_{\max }(\ell)+\frac{\pi}{4 \ell}\right\} .
\end{gathered}
$$


We define the functions:

$$
\begin{aligned}
g(k, \ell):= & g_{-}(k, \ell) e^{-4 \alpha_{-} \sqrt{1-k^{2}} \ell}+g_{+}(k, \ell) e^{-4 \alpha_{+} \sqrt{1-k^{2}} \ell} \\
& +g_{-}(k, \ell) g_{+}(k, \ell) e^{-4\left(\alpha_{-}+\alpha_{+}\right) \sqrt{1-k^{2}} \ell} \\
g_{-}(k, \ell):= & -\frac{\mathrm{i} k}{\sqrt{1-k^{2}}} \cdot \frac{a_{1}(k)}{a_{2}(k)\left(a_{2}(-k) b_{1}(k)-b_{2}(-k) a_{1}(k) e^{-4 \alpha_{-} \sqrt{1-k^{2}} \ell}\right)}, \\
g_{+}(k, \ell):= & -\frac{\mathrm{i} k}{\sqrt{1-k^{2}}} \cdot \frac{b_{4}(k)}{b_{3}(k)\left(b_{3}(-k) a_{4}(k)-a_{3}(-k) b_{4}(k) e^{-4 \alpha_{+} \sqrt{1-k^{2}} \ell}\right)}, \\
G(k, l):= & -\frac{\mathrm{i}}{4 \ell} \ln (1+g(k, \ell)), \\
L(k, \ell):= & \ell^{-1} h(k)+G(k, \ell), \quad L_{n}(z, \ell):=L\left(z+\varkappa_{n}(\ell), \ell\right)
\end{aligned}
$$

where the branch of the logarithm is fixed by the condition $\ln 1=0$.

Our main results are as follows.

Theorem 2.1. Assume that conditions (2.7), (2.8) are satisfied. Then for sufficiently large $\ell$ the operator $\mathcal{H}_{\ell}$ possesses exactly $N(\ell)$ eigenvalues/resonances $\lambda=k_{n}^{2}(\ell), n \in M_{\ell}$, in the domain $\Pi_{\ell}$. Each quantity $k_{n}(\ell)$ is represented by the limit

$$
k_{n}(\ell)=\varkappa_{n}(\ell)+\lim _{m \rightarrow+\infty} L_{n}^{[m]}(0, \ell)
$$

and the sums of the series

$$
k_{n}(\ell)=\varkappa_{n}(\ell)+\sum_{m=1}^{\infty} \frac{1}{m !} \frac{d^{m-1} L^{m}}{d k^{m-1}}\left(\varkappa_{n}(\ell), \ell\right)=\sum_{m=1}^{\infty} \frac{1}{m !} \frac{d^{m-1}\left(\varkappa_{n}+L\right)^{m}}{d k^{m-1}}(0, \ell) .
$$

Both series converges absolutely and uniformly in $\ell^{-1}$. The estimates hold:

$$
\begin{aligned}
\left|k_{n}(\ell)-\varkappa_{n}(\ell)-L_{n}^{[m]}(0, \ell)\right| \leqslant C_{0}\left(\frac{M+1}{\rho \ell}\right)^{m+1}, & \\
\left|k_{n}(\ell)-\varkappa_{n}(\ell)-\sum_{m=1}^{p} \frac{1}{m !} \frac{d^{m-1} L^{m}}{d k^{m-1}}\left(\varkappa_{n}(\ell), \ell\right)\right| & \leqslant \frac{1}{(p+1) !} \max _{k \in \Omega_{\ell}}\left|\frac{d^{p} L^{p+1}}{d k^{p}}(k, \ell)\right| \\
& \leqslant \frac{|\partial \Omega|}{2 \pi(p+1)}\left(\frac{M+1}{\rho \ell}\right)^{p+1}, \\
\left|k_{n}(\ell)-\sum_{m=1}^{p} \frac{1}{m !} \frac{d^{m-1}\left(\varkappa_{n}(\ell)+L\right)^{m}}{d k^{m-1}}(0, \ell)\right| & \leqslant \frac{1}{(p+1) !} \max _{k \in \overline{\Omega_{\ell}}}\left|\frac{d^{p}\left(\varkappa_{n}+L\right)^{m}}{d k^{p}}(k, \ell)\right| \\
& \leqslant \frac{|\partial \Omega|}{2 \pi(p+1)}\left(\frac{M+\beta+1}{\rho \ell}\right)^{p+1},
\end{aligned}
$$

where $C_{0}$ is some constant independent of $\ell, m$ and $n$.

We denote:

$$
P_{n}(z, \ell):=\left(1-\ell^{-1} \frac{h\left(z+K_{n}(\ell)\right)-h\left(K_{n}(\ell)\right)}{z}\right)^{-1} G\left(z+K_{n}(\ell), \ell\right) .
$$


Theorem 2.2. Assume that conditions (2.7), (2.8) are satisfied. Then the eigenvalues/resonances $\lambda=k_{n}^{2}(\ell), n \in M_{\ell}$, of the operator $\mathcal{H}_{\ell}$ in the domain $\Pi_{\ell}$ described in Theorem 2.1, satisfy the representations:

$$
\begin{aligned}
& k_{n}(\ell)=K_{n}(\ell)+\lim _{m \rightarrow+\infty} P_{n}^{[m]}(0, \ell), \\
& k_{n}(\ell)=K_{n}(\ell)+\sum_{m=1}^{\infty} \frac{1}{m !} \frac{d^{m-1} P_{n}^{m}}{d z^{m-1}}(0, \ell) .
\end{aligned}
$$

The latter series converges absolutely and uniformly in $\ell^{-1}$. The estimates hold:

$$
\begin{aligned}
& \left|k_{n}(\ell)-K_{n}(\ell)-P_{n}^{[m]}(0, \ell)\right| \leqslant C_{1} C_{2}^{m+1} e^{-4(m+1) \alpha \kappa \ell}, \\
& \left|k_{n}(\ell)-K_{n}(\ell)-\sum_{m=1}^{p} \frac{1}{m !} \frac{d^{m-1} P_{n}^{m}}{d z^{m-1}}(0, \ell)\right| \leqslant \frac{|\partial \Omega|}{2 \pi(p+1)} C_{2}^{p+1} e^{-4(p+1) \alpha \kappa \ell},
\end{aligned}
$$

where $C_{1}, C_{2}$ are some constants independent of $\ell, p, m$ and $n$, while the constant $\kappa$ is determined by the identity:

$$
\kappa:=\min _{k \in \bar{\Omega}} \operatorname{Re} \sqrt{1-k^{2}}>0 .
$$

\section{Discussion OF THE RESUlts}

Let us discuss the model and the main results. The potential $V_{\ell}$ is formed by four distant perturbations each being described by one of the potentials $V_{i}, i=1, \ldots, 4$. The potentials are separated by the distances of order $\ell$, see Figure 2. The final potential $V_{\ell}$ vanishes as $x<-\left(\alpha_{-}+1\right) \ell-x_{0}$ and $x>\left(\alpha_{+}+1\right) \ell+x_{0}$. This ensures identity (2.9). The main feature of the potential is that it vanishes also as $-\ell+x_{0}<x<\ell-x_{0}$. Exactly the latter generates the series of the resonances/eigenvalues $\lambda=k_{n}^{2}(\ell), n \in M_{\ell}$; a number $\lambda$ is a resonance if $\operatorname{Im} k_{n}(\ell) \leqslant 0$ and is an eigenvalue if $\operatorname{Im} k_{n}(\ell)>0$. We stress that the eigenvalues can emerge since the potential $V_{\ell}$ can be non-real and this makes the operator $\mathcal{H}_{\ell}$ non-self-adjoint. If the potential $V_{\ell}$ and the operator $\mathcal{H}_{\ell}$ is self-adjoint, the numbers $k_{n}(\ell)$ can correspond to the resonances only.

The mechanism of emergence of such resonances/eigenvalues is in general the same as in works [6], 12], [13]; from a physical point of view, there is a pronounced analogy with the Fabry-Perót interferometer. Namely, non-trivial solutions of equation (2.2) oscillate in the zone $-\ell+x_{0}<x<\ell-x_{0}$, where the potential $V_{\ell}$ vanishes and are exponentially small in neighbouring zones, $-\left(2 \alpha_{-}+1\right) \ell+x_{0}<x<-\ell-x_{0}$ and $\ell+x_{0}<x<\left(2 \alpha_{+}+1\right) \ell-x_{0}$, where the potential is equal to one. This is why, for large $\ell$, in the middle zone $-\ell+x_{0}<x<\ell-x_{0}$, these non-trivial solutions are approximated by the eigenfunctions of the Dirichlet problem for the operator $-\frac{d^{2}}{d x^{2}}$ on the segment $[-\ell, \ell]$. The eigenvalues of the latter operator are exactly the number $\varkappa_{n}^{2}(\ell)$ and this explains the presence of these numbers as the leading terms in identities (2.21), 2.22).

In works [6], [12], 13], the resonances/eigenvalues emerged along some segment in the essential spectrum; for the most of them, the distances to this segment were of order $\ell^{-1}$. The distances between the emerging resonances/eigenvalues were also of order $\ell^{-1}$ and as the parameter $\ell$ increased, their total number increased as well and they accumulated along the reference segment. It should be noted that a rigorous analysis of the location of the emerging eigenvalues/resonances was made only in [13], [12]; it was proved that there existed some segment in the essential spectrum, along which the emergence going.

In our case the situation is similar but there is a pair of principal differences. The first of them that the segment in the essential spectrum, along which the resonances emerge and accumulate, can be chose apriori and rather arbitrary: this is the segment $\left[\beta_{-}, \beta_{+}\right]$defined in terms of the variable $k$; in terms of the variable $\lambda$ it reads as $\Lambda:=\left\{\lambda: \lambda=k^{2}, k \in\left[\beta_{-}, \beta_{+}\right]\right\}$. At that, 
conditions (2.7), 2.8) do not influence the emergence of the resonances/eigenvalues in the sense that if these conditions fail, the spectral picture is similar to that in works [12, [13] and the emergence occurs along the segment $\Lambda$. Conditions (2.7), (2.8) ensure the second important difference: the imaginary parts of all quantities $k_{n}(\ell)$ are exponentially small in $\ell$. Let us dwell on the second difference in more details.

Theorem 2.1 states the emergence and accumulation of the resonances/eigenvalues. Namely, it states the existence of the corresponding set of numbers $k_{n}(\ell), n \in M_{\ell}$ along the given segment $\left[\beta_{-}, \beta_{+}\right]$. These quantities are represented by either limits (2.21) or by series (2.22). Estimates (2.23), (2.24), 2.25) show the sharpness of approximating $k_{n}$ by the terms of the sequence in (2.21) and by the partial sums of the series in (2.22). In particular, it follows from estimate (2.24) that the quantities $k_{n}(\ell)$ are located in neighbourhoods of the numbers $\varkappa_{n}(\ell)$ and the sizes of these neighbourhoods are of order $\ell^{-1}$. At the same time, formulae (2.21), 2.22 do not give an explicit answer how the imaginary parts of the quantities $k_{n}(\ell)$ are small since in fact, one has to analyse the imaginary parts of each term in series $(2.22)$ being power in $\ell^{-1}$. This issue is resolved effectively in Theorem 2.2. Namely, here we provide representations (2.27), (2.28) for the differences $k_{n}(\ell)-K_{n}(\ell)$, where $K_{n}$ are real roots of equation (2.11); these representations are similar to 2.21), 2.22). The function $P_{n}$ is exponentially small for large $\ell$ thanks to the factor $G$, since it follows from (2.18), 2.20) that the functions $G$ and $P_{n}$ satisfy the estimates

$$
|G(k, \ell)| \leqslant C \ell^{-1} e^{-4 \alpha \kappa \ell}, \quad\left|P_{n}(k, \ell)\right| \leqslant C \ell^{-1} e^{-4 \alpha \kappa \ell},
$$

where $C$ are some constants independent of $k, \ell$ and $n$. Similar estimates hold for all derivatives of these functions. In view of inequalities (2.30) this means that all terms in series (2.28) are exponentially small in $\ell$. Therefore, the numbers $k_{n}(\ell)$ accumulate along the segment $\left[\beta_{-}, \beta_{+}\right]$ at exponentially small distances, while the mutual distances between these numbers are of order $\ell^{-1}$. This implies that the imaginary parts of the quantities $k_{n}(\ell)$ are exponentially small. In particular, the identities hold:

$$
\begin{aligned}
& G(k, \ell)=-\frac{\mathrm{i}}{4 \ell} g(k, \ell)+O\left(\ell^{-1}|g(k, \ell)|^{2}\right)=g_{0}(k, \ell)+O\left(e^{-8 \alpha \sqrt{1-k^{2}} \ell}\right), \\
& g_{0}(k, \ell):=-\frac{k}{4 \ell \sqrt{1-k^{2}}}\left(\frac{a_{1}(k) e^{-4 \alpha_{-} \sqrt{1-k^{2} \ell}}}{a_{2}(k) a_{2}(-k) b_{1}(k)}+\frac{b_{4}(k) e^{-4 \alpha_{+} \sqrt{1-k^{2} \ell}}}{b_{3}(k) b_{3}(-k) a_{4}(k)}\right) .
\end{aligned}
$$

Hence, by estimate 2.30 with $p$ large enough and fixed by the condition

$$
(p+1) \kappa>2 \sqrt{1-\beta^{2}},
$$

we get that

$$
k_{n}(\ell)=K_{n}(\ell)+g_{0}\left(K_{n}(\ell), \ell\right)+O\left(e^{-8 \alpha \sqrt{1-K_{n}^{2}(\ell)} \ell}\right),
$$

where the estimate for the error term is uniform in $\ell$ and $n$. By the definition of the function $g_{0}$, the second term in the right hand side of the above identity is exponentially small for large $\ell$.

It should be stressed that conditions (2.7), (2.8) are not strict and can be easily realized. For instance, condition (2.8) holds immediately once the potential $V_{\ell}$ is partially real, namely, if the potentials $V_{2}$ and $V_{3}$ are real-valued. In this case we obviously have the identities

$$
a_{2}(-k)=\overline{a_{2}(k)}, \quad b_{3}(-k)=\overline{b_{3}(k)},
$$

which ensure condition (2.8) for all real values of $k$. Condition (2.7) can be also easily satisfied for arbitrary potentials $V_{i}$ by choosing an appropriate segment $\left[\beta_{-}, \beta_{+}\right]$: the functions $a_{i}, b_{i}$ are holomorphic, see the fifth section, and this is why can not vanish identically.

Assume that the function $f$ satisfies condition $(2.8)$ on some segment $\left[\beta_{-}, \beta_{+}\right]$, while on a large segment $\left[\tilde{\beta}_{-}, \tilde{\beta}_{+}\right]$outside $\left[\beta_{-}, \beta_{+}\right]$this condition fails. Then it can be shown that in this case the operator $\mathcal{H}_{\ell}$ possesses a series of resonances/eigenvalues with exponential asymptotics 
accumulating along the segment $\left[\beta_{-}, \beta_{+}\right]$described in Theorems 2.1, 2.2 and also a series of resonances/eigenvalues accumulating along the set $\left[\tilde{\beta}_{-}, \tilde{\beta}_{+}\right] \backslash\left[\beta_{-}, \beta_{+}\right]$and possessing a power in $\ell^{-1}$ behavior described in works [12], [13]. Thus, it is possible to realize a situation, when an operator with distant perturbations possesses unboundedly many resonances/eigenvalues with both power and exponentially small imaginary parts.

We also mention that the range of values of $\ell$, for which Lemma 2.1 and Theorems 2.1, 2.2 hold can be given rather explicitly. Namely, representation (2.13) and estimates (2.15) are true as

$$
\ell>M^{\prime}
$$

The convergence of the first series in (2.14) and the validity of estimates (2.16) are guaranteed by the inequality $\rho \ell>M$. The second series in (2.14) and estimates (2.17) are true as $\rho \ell>$ $M+\beta+1$. All these conditions obviously follow from estimates (2.15), (2.16), (2.17). A similar situation is the statement of Theorem 2.1, here the range of the values of $\ell$ is determined by the inequalities

$$
|L(k, \ell)| \leqslant(M+1) \ell^{-1}, \quad k \in \bar{\Omega}, \quad \rho \ell>M+1,
$$

This ensures (2.21), the first identity in $(2.22)$ and estimates $(2.23),(2.24)$. The second inequality in 2.22 and estimates 2.25 hold once we assume additionally that $\rho \ell>M+\beta+1$. And finally, the statement of Theorem 2.2 is true for $\ell$ obeying the inequalities

$$
\ell>M^{\prime}, \quad|G(k, \ell)| \leqslant C_{2} e^{-4 \alpha \kappa \ell}<1-M^{\prime} \ell^{-1}, \quad \rho \ell>C_{2} .
$$

\section{EquATION FOR RESONANCES AND EIGENVALUES}

Non-trivial solutions of equation $(2.2)$ obeying conditions $(2.3)$ can be easily constructed in terms of the functions $X_{i}$. Namely, if $\psi$ is such non-trivial solution, then $c_{ \pm} \neq 0$ since otherwise the function $\psi$ would be identically zero for $\pm x>x_{0}$ and this would imply immediately that $\psi$ vanishes identically on $\mathbb{R}$. This is why without loss of generality we suppose that $c_{-}=1$. In view of the definition of the potnetial $V_{\ell}$ we hence conclude that

$$
\psi(x, k, \ell)=X_{1}\left(x+\left(2 \alpha_{-}+1\right) \ell, k\right), \quad x \leqslant-\left(\alpha_{-}+1\right) \ell .
$$

As $x>-\left(\alpha_{-}+1\right) \ell+x_{0}$, by $(2.5)$ we have:

$$
X_{1}\left(x+\left(2 \alpha_{-}+1\right) \ell, k\right)=a_{1}(k) e^{-\left(2 \alpha_{-}+1\right) \sqrt{1-k^{2}} \ell} e^{-\sqrt{1-k^{2}} x}+b_{1}(k) e^{\left(2 \alpha_{-}+1\right) \sqrt{1-k^{2}} \ell} e^{\sqrt{1-k^{2}} x} .
$$

Let $k \neq 0$. Then by identities (2.6) the functions $X_{2}(x, k)$ and $X_{2}(x,-k)$ are linearly independent and this is why they form a fundamental system of solutions for equation (2.4). This is why by definition (2.1) of the potential $V_{\ell}$, as $-\left(2 \alpha_{-}+1\right)<\ell<x<0$, the function $\psi$ reads as

$$
\psi(x, k, \ell)=A_{2}(k, \ell) X_{2}(x+\ell, k)+B_{2}(k, \ell) X_{2}(x+\ell,-k),
$$

where $A_{2}=A_{2}(k, \ell)$ and $B_{2}=B_{2}(k, \ell)$ are some constants. According (2.6), for $x<-\ell-x_{0}$ we get:

$$
X_{2}(x+\ell, \pm k)=a_{2}( \pm k) e^{-\sqrt{1-k^{2}} \ell} e^{-\sqrt{1-k^{2}} x}+b_{2}( \pm k) e^{\sqrt{1-k^{2}} \ell} e^{\sqrt{1-k^{2}} x} .
$$

Substituting these relations into (4.3) and comparing the result with (4.2), we see that a required smoothness of the function $\psi$ is equivalent to the following equations:

$$
\begin{aligned}
& a_{1}(k) e^{-\left(2 \alpha_{-}+1\right) \sqrt{1-k^{2}} \ell}=\left(a_{2}(k) A_{2}(k, \ell)+a_{2}(-k) B_{2}(k, \ell)\right) e^{-\sqrt{1-k^{2}} \ell}, \\
& b_{1}(k) e^{\left(2 \alpha_{-}+1\right) \sqrt{1-k^{2}} \ell}=\left(b_{2}(k) A_{2}(k, \ell)+b_{2}(-k) B_{2}(k, \ell)\right) e^{\sqrt{1-k^{2}} \ell} .
\end{aligned}
$$

These relations define uniquely the constants $A_{2}(k)$ and $B_{2}(k)$ under the condition

$$
\left|\begin{array}{cc}
a_{2}(k) & a_{2}(-k) \\
b_{2}(k) & b_{2}(-k)
\end{array}\right| \neq 0
$$


Let us prove that this inequality indeed holds. We first observe that by equation (2.4) the Wronskian of the functions $X_{2}(x,-k)$ and $X_{2}(x, k)$ is non-zero and is independent of $x$. Calculating this Wronskian for sufficiently large positive and negative $x$ on the base of relations (2.6), we obtain:

$$
\begin{aligned}
2 i k & =\sqrt{1-k^{2}}\left(\left|\begin{array}{rr}
a_{2}(-k) & b_{2}(k) \\
-a_{2}(-k) & b_{2}(k)
\end{array}\right|-\left|\begin{array}{rr}
a_{2}(k) & b_{2}(-k) \\
-a_{2}(k) & b_{2}(-k)
\end{array}\right|\right) \\
& =-2 \sqrt{1-k^{2}}\left|\begin{array}{ll}
a_{2}(k) & a_{2}(-k) \\
b_{2}(k) & b_{2}(-k)
\end{array}\right|
\end{aligned}
$$

which implies inequality (4.6). Now the constants $A_{2}(k, \ell)$ and $B_{2}(k, \ell)$ are determined uniquely by the formulae

$$
\begin{aligned}
& A_{2}(k, \ell):=\mathrm{i} \frac{\sqrt{1-k^{2}}}{k}\left(b_{2}(-k) a_{1}(k) e^{-2 \alpha_{-} \sqrt{1-k^{2}} \ell}-a_{2}(-k) b_{1}(k) e^{2 \alpha_{-} \sqrt{1-k^{2}} \ell}\right), \\
& B_{2}(k, \ell):=\mathrm{i} \frac{\sqrt{1-k^{2}}}{k}\left(a_{2}(k) b_{1}(k) e^{2 \alpha_{-} \sqrt{1-k^{2}} \ell}-b_{2}(k) a_{1}(k) e^{-2 \alpha_{-} \sqrt{1-k^{2}} \ell}\right) .
\end{aligned}
$$

We also note that according formulae (2.6), as $-\ell+x_{0}<x<0$, function (4.3) casts into the form:

$$
\psi(x, k, \ell)=A_{2}(k, \ell) e^{\mathrm{i} k \ell} e^{\mathrm{i} k x}+B_{2}(k, \ell) e^{-\mathrm{i} k \ell} e^{-\mathrm{i} k x} .
$$

Let us find the function $\psi(x)$ for $x>0$. According $(2.3)$ and the definition of the potential $V_{\ell}$, for $x>\left(2 \alpha_{+}+1\right) \ell+x_{0}$ the function $\psi$ should read as

$$
\psi(x, k)=c_{+} e^{i k x}, \quad c_{+} \neq 0,
$$

and this is why, by the definition of the function $X_{4}$, similar to 4.1) we conclude immediately that

$$
\psi(x, k, \ell)=c_{+} X_{4}\left(x-\left(2 \alpha_{+}+1\right) \ell, k\right) .
$$

As in 4.3, 4.4, 4.5, by 2.5 this implies that

$$
\psi(x)=c_{+}\left(A_{3}(k, \ell) X_{3}(x-\ell, k)+B_{3}(k, \ell) X_{3}(x-\ell,-k)\right) \quad \text { as } \quad 0<x<\alpha_{+} \ell,
$$

where $A_{3}=A_{3}(k, \ell)$ and $B_{3}=B_{3}(k, \ell)$ is a solution to a system of linear equations:

$$
\begin{aligned}
& a_{4}(k) e^{\left(2 \alpha_{+}+1\right) \sqrt{1-k^{2}} \ell}=\left(a_{3}(k) A_{3}(k, \ell)+a_{3}(-k) B_{3}(k, \ell)\right) e^{\sqrt{1-k^{2}} \ell}, \\
& b_{4}(k) e^{-\left(2 \alpha_{+}+1\right) \sqrt{1-k^{2}} \ell}=\left(b_{3}(k) A_{3}(k, \ell)+b_{3}(-k) B_{3}(k, \ell)\right) e^{-\sqrt{1-k^{2}} \ell} .
\end{aligned}
$$

An analogue of identity (4.7) here reads as

$$
\begin{aligned}
-2 i k & =\sqrt{1-k^{2}}\left(\left|\begin{array}{rr}
a_{3}(-k) & b_{3}(k) \\
-a_{3}(-k) & b_{3}(k)
\end{array}\right|-\left|\begin{array}{rr}
a_{3}(k) & b_{3}(-k) \\
-a_{3}(k) & b_{3}(-k)
\end{array}\right|\right) \\
& =-2 \sqrt{1-k^{2}}\left|\begin{array}{ll}
a_{3}(k) & a_{3}(-k) \\
b_{3}(k) & b_{3}(-k)
\end{array}\right|,
\end{aligned}
$$

while the formulae for the coefficients $A_{3}, B_{3}$ are as follows:

$$
\begin{aligned}
& A_{3}(k):=-\mathrm{i} \frac{\sqrt{1-k^{2}}}{k}\left(b_{3}(-k) a_{4}(k) e^{2 \alpha_{+} \sqrt{1-k^{2}} \ell}-a_{3}(-k) b_{4}(k) e^{-2 \alpha_{+} \sqrt{1-k^{2}} \ell}\right), \\
& B_{3}(k):=-\mathrm{i} \frac{\sqrt{1-k^{2}}}{k}\left(a_{3}(k) b_{4}(k) e^{-2 \alpha_{+} \sqrt{1-k^{2}} \ell}-b_{3}(k) a_{4}(k) e^{2 \alpha_{+} \sqrt{1-k^{2}} \ell}\right) .
\end{aligned}
$$

For $0<x<\ell-x_{0}$ the function $\psi$ becomes

$$
\psi(x, k, \ell)=c_{+}\left(A_{3}(k, \ell) e^{\mathrm{i} k \ell} e^{-\mathrm{i} k x}+B_{3}(k, \ell) e^{-\mathrm{i} k \ell} e^{\mathrm{i} k x}\right) .
$$


At the point $x=0$ the function $\psi$ should be continuous and should have a continuous derivative. These conditions are checked on the base of identities (4.9), (4.12). It is clear that this determines a constant $c_{+}$in 4.12 and produces an additional equation for the functions $A_{2}, B_{2}, A_{3}, B_{3}$ :

$$
A_{2}(k, \ell) A_{3}(k, \ell) e^{2 \mathrm{i} k \ell}=B_{2}(k, \ell) B_{3}(k, \ell) e^{-2 \mathrm{i} k \ell} .
$$

The latter equation determines the resonances and eigenvalues of the operator $\mathcal{H}_{\ell}$. Although this equation has been obtained under the assumption $k \neq 0$, it is appropriate also for finding the resonances and eigenvalues at the point $k=0$. Then formulae (4.9), (4.12) are to be treated in the sense of the limit as $k \rightarrow 0$.

The solvability of the obtained equation and the behavior of its roots for large values of $\ell$ will be done in the next section.

\section{StUdy OF EQUATION FOR EIGENVALUES AND RESONANCES}

In the present section we study equation 4.13) and prove Theorems 2.1, 2.2. We first substitute expressions (4.8), 4.11) into this equation and rewrite it as follows:

$$
e^{4 \mathrm{i} k \ell}=F(k, \ell)
$$

where we have denoted

$$
F(k, \ell):=\frac{a_{2}(k) b_{1}(k)-b_{2}(k) a_{1}(k) e^{-4 \alpha_{-} \sqrt{1-k^{2}} \ell}}{a_{2}(-k) b_{1}(k)-b_{2}(-k) a_{1}(k) e^{-4 \alpha_{-} \sqrt{1-k^{2}} \ell}} \cdot \frac{b_{3}(k) a_{4}(k)-a_{3}(k) b_{4}(k) e^{-4 \alpha_{+} \sqrt{1-k^{2}} \ell}}{b_{3}(-k) a_{4}(k)-a_{3}(-k) b_{4}(k) e^{-4 \alpha_{+} \sqrt{1-k^{2} \ell}}} .
$$

In what follows we shall make use of the smoothness of the functions $a_{i}$ and $b_{i}$ with respect to $k$. Rewriting equations (2.4) and conditions (2.5) to Volterra integral equations in the standard way, it is easy to make sure that the functions $a_{i}$ and $b_{i}$ are holomorphic in $k \in \Xi$. Moreover, by the definition of the function $k \mapsto \sqrt{1-k^{2}}$ we have

$$
\operatorname{Re} \sqrt{1-k^{2}}>0, \quad k \in \Xi .
$$

Let $\Omega$ be some fixed open neighbourhood of the segment $\left[\beta_{-}, \beta_{+}\right]$in the complex plane such that $\bar{\Omega} \subset \Xi$. Then it is clear that the neighbourhood $\Omega$ is separated from the boundaries of the set $\Xi$ by a positive distance that implies the positivity of the constant $\kappa$ in (2.31).

It follows from the holomorphy of $a_{j}$ and $b_{j}$ in $k \in \bar{\Omega}$ and condition 2.7 that the function $f(k)$ is holomorphic in $k \in \bar{\Omega}$ if the neighbourhood $\Omega$ is not too large. In view of inequality 2.31 we also conclude that the functions $F(k, \ell)$ and $g(k, \ell)$ are holomorphic in $k \in \bar{\Omega}$ and uniform estimates hold:

$$
\begin{aligned}
& |g(k, \ell)| \leqslant C e^{-4 \alpha \sqrt{1-k^{2}} \ell} \leqslant C e^{-4 \alpha \kappa \ell}, \\
& \left|g^{\prime}(k, \ell)\right| \leqslant C \ell e^{-4 \alpha \sqrt{1-k^{2}} \ell} \leqslant C \ell e^{-4 \alpha \kappa \ell},
\end{aligned}
$$

where $C$ is some constant independent of $k$ and sufficiently large $\ell$. Moreover, by direct calculations on the base of relations (4.7), 4.10) we confirm the identity

$$
F(k, \ell)=f(k)+g(k, \ell)
$$

The latter identity allows us to rewrite equation (5.1) as

$$
e^{4 \mathrm{i} k \ell}=f(k)+g(k, \ell) .
$$

To study the latter equation in the domain $\Omega$, we first consider auxiliary equation (2.11).

Lemma 5.1. All roots of equation 2.11) located in $\Omega$ satisfy the estimate

$$
-\frac{1}{4 \ell} \ln \max _{k \in \bar{\Omega}}|f(k)| \leqslant \operatorname{Im} k \leqslant-\frac{1}{4 \ell} \ln \min _{k \in \bar{\Omega}}|f(k)| .
$$


All roots of equation (5.4) located in $\Omega$ satisfy the estimate

$$
-\frac{1}{4 \ell} \ln \max _{k \in \bar{\Omega}}|f(k)|-\frac{C e^{-4 \kappa \alpha \ell}}{4 \ell} \leqslant \operatorname{Im} k \leqslant-\frac{1}{4 \ell} \ln \min _{k \in \bar{\Omega}}|f(k)|+\frac{C e^{-4 \kappa \alpha \ell}}{4 \ell},
$$

where $C$ is the constant from 5.2).

Proof. If $k$ is a root of equation 2.11), then an elementary apriori estimate

$$
\min _{k \in \bar{\Omega}}|f(k)| \leqslant\left|e^{\mathrm{i} k \ell}\right| \leqslant \max _{k \in \bar{\Omega}}|f(k)|
$$

holds. And since $\left|e^{4 \mathrm{i} k \ell}\right|=e^{-4 \ell \operatorname{Im} k}$, we are led immediately to the statement of the lemma on the roots of equation (2.11). The desired estimate for the roots of equation (2.11) can be proved in the same way, one just need to employ estimate (2.31). The proof is complete.

Remark 5.1. Condition (2.8) yields that

$$
\min _{k \in \bar{\Omega}}|f(k)| \leqslant 1 \leqslant \max _{k \in \bar{\Omega}}|f(k)| .
$$

This is why the left hand side in (5.5) is non-positive, while the right hand side is non-negative.

In view of condition (2.8) we suppose that the domain $\Omega$ is chosen so that

$$
|f(k)| \geqslant c_{0}>0 \quad \text { as } \quad k \in \bar{\Omega} .
$$

This allows us to define the function $h(k)$ by formula 2.10$)$ and a branch of the logarithm in the definition of this function can be chosen so that the function $h$ becomes holomorphic in the domain $\bar{\Omega}$. For the mentioned choice of the function $h$, it is sufficient to employ an approach based on the notion of a complete analytic function, see, for instance, [4, Ch. 8, Sects. 4, 5]. We also mention that by (2.8) the function $h(k)$ is real-valued for $k \in\left[\beta_{-}, \beta_{+}\right]$.

Thanks to the definition of the function $h(k)$, the identity

$$
f(k)=e^{\mathrm{i} h(k)}
$$

holds and equation (2.11) turns out to be equivalent to the following one:

$$
k-\ell^{-1} h(k)=\varkappa_{n}(\ell), \quad n \in \mathbb{Z} .
$$

Since the function $h(k)$ is real for $k \in\left[\beta_{-}, \beta_{+}\right]$, its derivative is also real on this segment and is bounded uniformly owing to the smoothness of the function $h$. This is why, for sufficiently large $\ell$,

$$
\frac{d}{d k}\left(k-\ell^{-1} h(k)\right)=1-\ell^{-1} h^{\prime}(k)>0 \quad \text { as } \quad k \in\left[\beta_{-}, \beta_{+}\right]
$$

and this is why the function in the left hand side of equation $(5.6)$ turns out to be monotonically increasing in $k \in\left[\beta_{-}, \beta_{+}\right]$. Its values cover the segment $I_{\ell}$ defined in (2.12); the end-points of this segment tend to $\beta_{-}$and $\beta_{+}$as $\ell \rightarrow+\infty$. It is clear that for large $\ell$ the segment $I_{\ell}$ contains a large number of the points $\varkappa_{n}(\ell)$ and to each such point, exactly one real root of equation (5.6) corresponds. We denote this root by $K_{n}=K_{n}(\ell)$, where $n \in \mathbb{M}_{\ell}$, and the set $\mathrm{M}_{\ell}$ is defined by formula 2.12 .

We denote

$$
\Omega_{\ell}:=\left\{k \in \Omega: \operatorname{dist}\left(k,\left[\beta_{-}, \beta_{+}\right]\right) \leqslant M \ell^{-1}\right\} .
$$

It follows from the definition of the sets $\Omega_{\ell}$ and $\Omega$ that for sufficiently large $\ell$ the inequality

$$
\operatorname{dist}\left(\partial \Omega, \Omega_{\ell}\right) \geqslant \rho
$$

holds true. It also follows from the definition of the segment $I_{\ell}$ that $I_{\ell} \subset \Omega_{\ell}$ and inequalities (5.5) yield that all roots of equation (2.11) with real parts in the segment $I_{\ell}$ are located in the set $\Omega_{\ell}$. 
Lemma 5.2. For $\ell$ satisfying condition (3.1) and $n \in \mathbb{M}_{\ell}$, equation (5.6) has only real roots $K_{n}$ in the domain $\Omega_{\ell}$, which are represented by limit (2.13) and the sum of series (2.14). Both series converge absolutely and uniformly in $\ell^{-1}$. Estimates (2.15), (2.16) and (2.17) hold true.

Proof. For each $n \in \mathbb{M}_{\ell}$ we define a set:

$$
S_{n}(\ell):=\left\{k:\left|\operatorname{Re} k-\varkappa_{n}(\ell)\right| \leqslant M \ell^{-1},|\operatorname{Im} k| \leqslant M \ell^{-1}\right\} .
$$

The definition of the constant $M$ and Lemma 5.1 imply immediately that the sets $S_{n}(\ell)$ cover $\Omega_{\ell}$, that is, $\Omega_{\ell} \subset \bigcup_{n \in \mathbb{M}_{\ell}} S_{n}(\ell)$.

Equation (5.6) implies an apriori estimate for the roots $K_{n}$ :

$$
\left|K_{n}(\ell)-\varkappa_{n}(\ell)\right| \leqslant\left|\ell^{-1} h\left(K_{n}(\ell)\right)\right| \leqslant M \ell^{-1}
$$

and this is why the root $K_{n}$ is a point in the set $S_{n}(\ell)$.

We make the change of the variable $z:=k-\varkappa_{n}(\ell)$. Then, as the variable $k$ ranges over the set $S_{n}(\ell)$, a new variable $z$ ranges over the set $S_{0}(\ell)$. The estimates hold:

$$
\begin{array}{ll}
\left|\operatorname{Re} \ell^{-1} h\left(z+\varkappa_{n}(\ell)\right)\right| \leqslant M \ell^{-1}, \quad\left|\operatorname{Im} \ell^{-1} h\left(z+\varkappa_{n}(\ell)\right)\right| \leqslant M \ell^{-1}, & z \in S_{0}(\ell), \\
\left|\ell^{-1} h\left(z_{1}+\varkappa_{n}(\ell)\right)-\ell^{-1} h\left(z_{2}+\varkappa_{n}(\ell)\right)\right| \leqslant \ell^{-1} \max _{k \in \bar{\Omega}}\left|h^{\prime}(k)\right|\left|z_{2}-z_{1}\right|, & z_{1}, z_{2} \in S_{0}(\ell) .
\end{array}
$$

It follows from these estimates that the function $z \mapsto \ell^{-1} h\left(z+\varkappa_{n}(\ell)\right)$ maps a compact set $S_{0}(\ell)$ into itself and is a contracting mapping for $\ell$ obeying condition (3.1). This is why equation (5.6) has a unique solution in the set $S_{n}(\ell)$, which hence coincides with $K_{n}$. Representation (2.13) is an immediate corollary of the contracting mapping principle. Representations (2.14), estimates 2.15) and the first estimates for the remainders in 2.16), 2.17) can be proved in the same way how similar representations and estimates in Theorem 1 in [13. The second estimates for the remainders in (2.16), (2.17) follow from the standard estimates for the derivatives of holomorphic functions implied by the Cauchy integral formula and inequality (5.8):

$$
\begin{aligned}
& \max _{k \in \Omega_{\ell}}\left|\frac{d^{p} h^{p+1}}{d k^{p}}(k)\right| \leqslant \frac{p !|\partial \Omega|}{2 \pi}\left(\frac{M}{\rho}\right)^{p+1}, \\
& \max _{\overline{\Omega_{\ell}}}\left|\frac{d^{p}}{d k^{p}}\left(\varkappa_{n}(\ell)+h(k)\right)^{p+1}\right| \leqslant \frac{p !|\partial \Omega|}{2 \pi}\left(\frac{M+\beta+1}{\rho}\right)^{p+1} .
\end{aligned}
$$

The proof is complete.

Lemma 2.1 is an immediate corollary of the proven lemma.

We proceed to studying main equation (5.4) and we are going to prove Theorems 2.1, 2.2.

Proof of Theorem 2.1. We rewrite equation (5.4) as

$$
e^{4 \mathrm{i} k \ell-4 \mathrm{i} h(k)}=1+e^{-4 \mathrm{i} h(k)} g(k, \ell) .
$$

Since by estimate $(5.2)$ the function $g$ is exponentially small, the function $G(k, \ell)$ in 2.18 is well-defined, holomorphic in $k \in \Omega$, univalent and exponentially small according to an obvious estimate:

$$
|G(k, \ell)| \leqslant C \ell^{-1}\left|e^{-4 \mathrm{i} h(k)} g(k, \ell)\right| \leqslant C \ell^{-1} e^{-4 \alpha \kappa \ell},
$$

where $C$ is a constant independent of $\ell$ and $k$. This allows us to rewrite equation (5.10) as follows:

$$
k=\varkappa_{n}(\ell)+L(k, \ell), \quad n \in \mathbb{Z},
$$

where $L$ is the function from 2.20). We are going to study the obtained equation reproducing in fact the ideas in the proof of Lemma 5.2.

The definition of the constant $M$ in (5.7) and estimate (5.11) yield that

$$
\max _{k \in \bar{\Omega}}|L(k, \ell)| \leqslant(M+1) \ell^{-1}
$$


for sufficiently large $\ell$. Estimates $(5.2),(5.3)$ definition (2.18) of the function $G$ also imply that

$$
\left|G^{\prime}(k, \ell)\right| \leqslant C e^{-4 \alpha \kappa \ell}, \quad k \in \Omega,
$$

where $C$ is some constant independent of $\ell$ and $k$.

We make the change of the variable $z=k-\varkappa_{n}(\ell)$. Similar to estimates (5.9), by employing inequalities (5.11), (5.13), (5.14) we confirm that

$$
\begin{array}{ll}
\left|\operatorname{Re} L_{n}(z, \ell)\right| \leqslant(M+1) \ell^{-1}, \quad\left|\operatorname{Im} L_{n}(z, \ell)\right| \leqslant(M+1) \ell^{-1}, & z \in S_{L}(\ell), \\
\left|L_{n}\left(z_{1}, \ell\right)-L_{n}\left(z_{2}, \ell\right)\right| \leqslant C \ell^{-1}\left|z_{2}-z_{1}\right|, & z_{1}, z_{2} \in S_{L}(\ell),
\end{array}
$$

where $C$ is a constant independent of $z_{1}, z_{2}$ and $\ell$, while the set $S_{L}(\ell)$ is of the form

$$
S_{L}(\ell):=\left\{z:|\operatorname{Re} z| \leqslant(M+1) \ell^{-1},|\operatorname{Im} z| \leqslant(M+1) \ell^{-1}\right\} .
$$

Therefore, the function $z \mapsto L_{n}(z, \ell)$ maps the set $S_{L}(\ell)$ into itself and is a contracting mapping. This implies immediately that equation (5.12) possesses exactly one root in $S_{L}(\ell)$, which is denoted by $k_{n}(\ell)$. According the contracting mapping principle, this root can be found as the limit of a corresponding sequence (2.21). And again similar to the proof of Theorem 1 in [13] we establish representations 2.22 , confirm the absolute uniform in $\ell^{-1}$ convergence of these series and prove inequalities (2.23) and the estimates for the remainders:

$$
\begin{aligned}
& \left|k_{n}(\ell)-\varkappa_{n}(\ell)-\sum_{m=1}^{p} \frac{1}{m !} \frac{d^{m-1} L^{m}}{d k^{m-1}}\left(\varkappa_{n}(\ell), \ell\right)\right| \leqslant \frac{1}{(p+1) !} \max _{k \in \Omega_{\ell}}\left|\frac{d^{p} L^{p+1}}{d k^{p}}(k, \ell)\right|, \\
& k_{n}(\ell)-\sum_{m=1}^{p} \frac{1}{m !} \frac{d^{m-1}\left(\varkappa_{n}(\ell)+L\right)^{m}}{d k^{m-1}}(0, \ell)\left|\leqslant \frac{1}{(p+1) !} \max _{k \in \overline{\Omega_{\ell}}}\right| \frac{d^{p}\left(\varkappa_{n}+L\right)^{m}}{d k^{p}}(k, \ell) \mid .
\end{aligned}
$$

The aforementioned standard estimates for the derivatives of holomorphic functions again allow to continue estimating and thanks to (5.8), (5.13) we get:

$$
\begin{aligned}
& \left|k_{n}(\ell)-\varkappa_{n}(\ell)-\sum_{m=1}^{p} \frac{1}{m !} \frac{d^{m-1} L^{m}}{d k^{m-1}}\left(\varkappa_{n}(\ell), \ell\right)\right| \leqslant \frac{|\partial \Omega|}{2 \pi(p+1)}\left(\frac{M+1}{\rho \ell}\right)^{p+1}, \\
& k_{n}(\ell)-\sum_{m=1}^{p} \frac{1}{m !} \frac{d^{m-1}\left(\varkappa_{n}(\ell)+L\right)^{m}}{d k^{m-1}}(0, \ell) \mid \leqslant \frac{|\partial \Omega|}{2 \pi(p+1)}\left(\frac{M+\beta+1}{\rho \ell}\right)^{p+1},
\end{aligned}
$$

and this completes the proof of Theorem 2.1.

Now we are going to describe the differences $k_{n}(\ell)-K_{n}(\ell)$ and to prove Theorem 2.2 .

Proof of Theorem 2.2. We consider equation (5.12) and make the change $z=k-K_{n}(\ell)$. Then in view of equation (5.6), for a new unknown $z$ we get:

$$
z=\ell^{-1}\left(h\left(z+K_{n}(\ell)\right)-h\left(K_{n}(\ell)\right)\right)+G\left(z+K_{n}(\ell), \ell\right) .
$$

We rewrite this equation as follows:

$$
\left(1-\ell^{-1} \frac{h\left(z+K_{n}(\ell)\right)-h\left(K_{n}(\ell)\right)}{z}\right) z=G\left(z+K_{n}(\ell), \ell\right)
$$

which implies one more equation:

$$
z=P_{n}(z, \ell)
$$

where the function $P_{n}$ comes from (2.26). We define a set: $S:=\{z \in \mathbb{C}:|z| \leqslant \delta\}$, where $\delta$ is some sufficiently small fixed number. By the holomorphy of the function $h$ and inequalities (5.2), (5.3) we conclude that the function $P_{n}(\cdot, \ell)$ is holomorphic in $\bar{S}$ and obeys uniform estimates:

$$
\left|P_{n}(z, \ell)\right| \leqslant C e^{-4 \alpha \kappa \ell}, \quad\left|P_{n}^{\prime}(z, \ell)\right| \leqslant C \ell e^{-4 \alpha \kappa \ell}
$$


where $C$ is some constant independent of $k$ and sufficiently large $\ell$. Now, similar to estimates (5.9), it is easy to confirm that the function $z \mapsto P_{n}(z, \ell)$ maps the set $\bar{S}$ into itself and is a contracting mapping on this set. A corresponding fixed point, a root of equation (5.16), can be found as a limit of a corresponding sequence satisfying estimates (2.29) or as a sum of series similar to $(2.22$ with estimates for the remainders similar to $(5.15)$. This gives rise to representations (2.27), (2.28) for the roots $k_{n}(\ell)$ and the following estimates for the remainders:

$$
\left|k_{n}(\ell)-K_{n}(\ell)-\sum_{m=1}^{p} \frac{1}{m !} \frac{d^{m-1} P_{n}^{m}}{d z^{m-1}}(0, \ell)\right| \leqslant \frac{1}{(p+1) !} \max _{z \in \bar{S}}\left|\frac{d^{p} P_{n}^{p+1}}{d z^{p}}(z, \ell)\right|
$$

The latter estimate with $p=1$ and inequalities (5.17) imply that

$$
\left|k_{n}(\ell)-K_{n}(\ell)\right| \leqslant C \ell e^{-4 \alpha \kappa \ell},
$$

where $C$ is a constant independent of $\ell$ and $n$. The obtained estimate means that the roots of equation (5.16) are exponentially close to zero. This allows us to improve estimate (5.18) by reproducing once again an appropriate arguing from the proof of Theorem 1 in [13]:

$$
\left|k_{n}(\ell)-K_{n}(\ell)-\sum_{m=1}^{p} \frac{1}{m !} \frac{d^{m-1} P_{n}^{m}}{d z^{m-1}}(0, \ell)\right| \leqslant \frac{1}{(p+1) !} \max _{|z| \leqslant C \ell e^{-4 \alpha \kappa} \ell}\left|\frac{d^{p} P_{n}^{p+1}}{d z^{p}}(z, \ell)\right| .
$$

The function $P_{n}$ can be expressed in terms of the variable $k$ :

$$
P_{n}(z, \ell)=\left(1-\ell^{-1} \frac{h(k)-h\left(K_{n}(\ell)\right)}{k-K_{n}(\ell)}\right)^{-1} G(k, \ell) .
$$

This allows us to express the maximum with respect to $z$ in 5.19 via a corresponding maximum with respect to $k$ and to employ once again the aforementioned estimates for the derivatives of holomorphic functions. As a result, a final estimate for the remainder becomes 2.30). This completes the proof of Theorem 2.2 .

\section{BIBLIOGRAPHY}

1. D. Borisov, A. Golovina. On emergence of resonances from multiple eigenvalues of the Schrödinger operator in a cylinder with distant perturbations // Itogi Nauki i Tekhniki. Ser. Sovrem. Mat. Pril. Temat. Obz. 163, 3-14 (2019). (in Russian).

2. D.I. Borisov, A.M. Golovina. On the resolvents of periodic operators with distant perturbaions // Ufimskij Matem. Zhurn. 4:2, 65-73 (2012). [Ufa Math. J. 4:2, 65-73 (2012).]

3. A.M. Golovina. Spectrum of periodic elliptic operators with distant perturbations in space // Alg. Anal. 25:5, 32-60 (2013). [St. Petersburg Math. J. 25:5, 735-754 (2014).]

4. A.I. Markushevich. The theory of analytic functions. V. 2. Nauka, Moscow (1967). (in Russian).

5. T. Aktosun, M. Klaus, and Cornelis van der Mee. On the number of bound states for the onedimensional Schrödinger equation // J. Math. Phys. 39:9, 4249-4259 (1998).

6. F. Barra and P. Gaspard. Scattering in periodic systems: from resonances to band structure // J. Phys. A: Math. Gen. 32:18, 3357-3375 (1999).

7. D. Borisov and P. Exner. Exponential splitting of bound states in a waveguide with a pair of distant windows // J. Phys. A: Math. Gen. 37:10, 3411-3428 (2004).

8. D. Borisov and P. Exner. Distant perturbation asymptotics in window-coupled waveguides. I. The non-threshold case // J. Math. Phys. 47:11, 113502 (2006).

9. D. Borisov, P. Exner and A. Golovina. Tunneling resonances in systems without a classical trapping // J. Math. Phys. 54:1, 012102 (2013).

10. D.I. Borisov. Distant perturbations of the Laplacian in a multi-dimensional space // Ann. H. Poincaré. 8:7, 1371-1399 (2007).

11. D. Borisov. Asymptotic behaviour of the spectrum of a waveguide with distant perturbation // Math. Phys. Anal. Geom. 10:2, 155-196 (2007). 
12. D.I. Borisov, D.A. Zezyulin. Spacing gain and absorption in a simple $\mathcal{P} \mathcal{T}$-symmetric model: spectral singularities and ladders of eigenvalues and resonances // J. Phys. A. Math. Theor. 52:44, 445202 (2019).

13. D.I. Borisov, D.A. Zezyulin. Sequences of closely spaced resonances and eigenvalues for bipartite complex potentials // Appl. Math. Lett. 100, 106049 (2020).

14. D.I. Borisov, A.M. Golovina. On finitely many resonances emerging under distant perturbations in multi-dimensional cylinders // J. Math. Anal. Appl. (2021), to appear.

15. E.B. Davies. The twisting trick for double well Hamiltonians // Comm. Math. Phys. 85:3, 471479 (1982).

16. V. Graffi, E.M. Harrell II and H.J. Silverstone. The $\frac{1}{R}$ expansion for $\mathrm{H}_{2}^{+}$: analyticity, summability and asymptotics // Ann. Phys. 165:2, 441-483 (1985).

17. A.M. Golovina. On the resolvent of elliptic operators with distant perturbations in the space // Russ. J. Math. Phys. 19:2, 182-192 (2012).

18. A.M. Golovina. Discrete eigenvalues of periodic operators with distant perturbations // J. Math. Sci. 189:3, 342-364 (2013).

19. E.M. Harrell. Double wells // Comm. Math. Phys. 75:3, 239-261 (1980).

20. E.M. Harrell and M. Klaus. On the double-well problem for Dirac operators // Ann. de l'Inst. H. Poincaré. 38:2, 153-166 (1983).

21. R. Høegh-Krohn and M. Mebkhout. The $\frac{1}{r}$ expansion for the critical multiple well problem // Comm. Math. Phys. 91:1, 65-73 (1983).

22. M. Klaus. Some remarks on double-wells in one and three dimensions // Ann. de l'Inst. H. Poincaré. 34:4, 405-417 (1981).

23. M. Klaus and B. Simon. Binding of Schrödinger particles through conspiracy of potential wells // Ann. de l'Inst. H. Poincaré, sect. A. 30:2, 83-87 (1979).

24. F. Klopp. Resonances for large one-dimensional "ergodic" systems // Anal. PDE. 9:2, 259-352 (2016).

25. M. Zworski, S. Dyatlov. Mathematical theory of scattering resonances. Amer. Math. Soc., Providence, RI (2019).

Denis Ivanovich Borisov,

Institute of Mathematics,

Ufa Federal Research Center, RAS,

Chernyshevsky str. 112,

450008, Ufa, Russia

Bashkir State University,

Zaki Validi str. 32,

450000, Ufa, Russia

University of Hradec Králové,

Rokitanskeho, 62

50003, Hradec Králové, Czech Republic

E-mail: BorisovDI@yandex.ru

Maral Nurlanovna Konyrkulzhaeva,

Al-Farabi Kazakh National University,

al-Farabi av. 71,

050040, Almaty, Kazakhstan

International University of Information Technology,

Manas str. 8,

050000, Almaty, Kazakhstan

E-mail: maralkulzha@gmail.com 